\title{
Correction to: Seismic glitchness at Sos Enattos site: impact on intermediate black hole binaries detection efficiency
}

A. Allocca ${ }^{1,2}$, A. Berbellini ${ }^{3}$, L. Boschi ${ }^{3,4,5}$, E. Calloni ${ }^{1,2, a}$ (D) G. L. Cardello ${ }^{6,7}$,

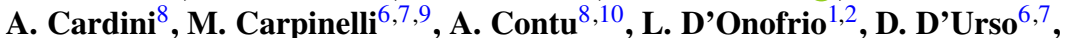

D. Dell'Aquila ${ }^{6,7}$, R. De Rosa ${ }^{1,2}$, L. Di Fiore ${ }^{2}$, M. Di Giovanni ${ }^{11,12,13}$, S. Di Pace ${ }^{14,15}$, L. Errico ${ }^{1,2}$, I. Fiori ${ }^{9}$, C. Giunchi ${ }^{11}$, A. Grado ${ }^{16}$, J. Harms ${ }^{12}$, E. Majorana ${ }^{14,15}$, V. Mangano ${ }^{14,15}$, M. Marsella ${ }^{14,15}$, C. Migoni $^{8}$, L. Naticchioni ${ }^{14,15}$, M. Olivieri ${ }^{3}$, G. Oggiano $^{6,7}$, F. Paoletti ${ }^{17}$, M. Punturo ${ }^{18}$, P. Puppo ${ }^{15}$, P. Rapagnani $^{14,15}$, F. Ricci $^{14,15}$, D. Rozza $^{6,7}$, G. Saccorotti ${ }^{11}$, V. Sequino ${ }^{1,2}$, V. Sipala ${ }^{6,7}$, I. Tosta E. Melo ${ }^{6,7}$, L. Trozzo ${ }^{2}$

${ }^{1}$ Universitá Federico II Napoli, 80126 Napoli, Italy

2 INFN - Sezione di Napoli, 80126 Napoli, Italy

3 INGV - Sezione di Bologna, 40128 Bologna, Italy

${ }^{4}$ Universitá Degli Studi di Padova, 35131 Padova, Italy

${ }^{5}$ Sorbonne Université, 75005 Paris, France

${ }^{6}$ Universitá Degli Studi di Sassari, 07100 Sassari, Italy

${ }^{7}$ INFN - Laboratori Nazionali del Sud, 95125 Catania, Italy

8 INFN - Sezione di Cagliari, 09042 Monserrato, CA, Italy

${ }^{9}$ European Gravitational Observatory, 56012 Cascina (PI), Italy

10 INAF - Osservatorio Astronomico di Cagliari, 09042 Cagliarii, Italy

${ }^{11}$ INGV - Sezione di Pisa, 56123 Pisa, Italy

12 Gran Sasso Science Institute, 67100 L'Aquila, Italy

13 INFN - Laboratori Nazionali del Gran Sasso, 67100 Assergi, AQ, Italy

14 Universitá di Roma La Sapienza, 00185 Roma, Italy

15 INFN - Sezione di Roma 1, 00185 Roma, Italy

16 INAF - Osservatorio Astronomico di Capodimonte, 80131 Napoli, Italy

17 INFN - Sezione di Pisa, 56127 Pisa, Italy

18 INFN - Sezione di Perugia 1, 06123 Perugia, Italy

(C) The Author(s), under exclusive licence to Società Italiana di Fisica and Springer-Verlag GmbH Germany, part of Springer Nature 2021

\section{Correction to: Eur. Phys. J. Plus (2021) 136:511}

$$
\text { https://doi.org/10.1140/epjp/s13360-021-01450-8 }
$$

The original version of this paper was inadvertently published with an incorrect author name for the author L. Naticchioni.

The correct name is: L. Naticchioni.

The original article has been corrected. We apologise for any inconvenience caused to our readers.

The original article can be found online at https://doi.org/10.1140/epjp/s13360-021-01450-8.

\footnotetext{
a e-mail: enrico.calloni@na.infn.it (corresponding author)
} 
The online version of the original article can be found under doi: https://doi.org/10.1140/ epjp/s13360-021-01450-8 